\title{
SOP Hirnnervenläsionen
}

Peter P. Urban

\section{Hirnnerven entspringen vor allem im Hirnstamm und übernehmen die Versorgung verschiedener Körperregionen mit ihren spezifischen Funktionen. Diese SOP beschreibt die häufigsten Ursachen von Läsionen, typische Untersuchungen und die spezielle Diagnostik der einzelnen Hirnnerven.}

\section{ÜBERSICHT / SYSTEMATIK}

Hirnnerven entspringen direkt aus dem Gehirn bzw. Hirnstamm. Eine Ausnahme bildet der N. accessorius, der aus dem Hirnstamm und dem zervikalen Rückenmark entsteht. Die ersten beiden Hirnnerven ( $\mathrm{N}$. olfactorius und $\mathrm{N}$. opticus) sind keine peripheren Nerven, sondern Teile des Gehirns, sie werden traditionell als Hirnnerven bezeichnet.

Ein Hirnnerv kann alle Faserqualitäten führen: somatomotorische, somatosensible und vegetative Fasern. Die Hirnnerven versorgen den Kopf, den Hals und mit parasympathischen Fasern die Organe im Rumpf. Hirnnervenläsionen (III bis XII) können durch eine Schädigung im Kerngebiet (nukleär), der Nervenfasern im Hirnstamm (faszikulär) oder extraaxial entstehen.

\section{Allgemeines Vorgehen zur Diagnose}

Eine Zusammenfassung gibt $>$ Abb. 1.

- Topodiagnose: Hirnnervenläsion vs. pseudo-peripherneurogene Läsion. Motorische Hirnnervenläsionen können durch Läsionen des ZNS (supranukleäre Läsion), neuromuskuläre Übertragungsstörungen und Myopathien imitiert werden. Bei motorischen oder afferenten Neuronen der Hirnnerven III-XII kann eine Läsion des Kerngebietes (nukleäre Läsion) oder der Nervenfasern im Hirnstamm (faszikuläre Läsion) klinisch und elektrophysiologisch nicht von einer weiter peripher gelegenen unterschieden werden, da es sich um das identische Axon handelt. Insofern kann einer „peripheren“ Hirnnervenläsion eine Hirnstammschädigung zugrunde liegen.

- zeitliche Dynamik des Auftretens (akut, subakut, chronisch) der Symptomatik

- Differenzierung zwischen unilateralen, bilateralen (z. B. Guillain-Barré-Syndrom, Miller-Fisher-Syndrom) oder multiplen Hirnnervenausfällen (z. B. Läsion des
N. IX, X, XI als Foramen-jugulare-Syndrom oder des N. V, III, IV, VI als Sinus-cavernosus-Syndrom)

- zusätzliche Symptome: z. B. Schmerzen der lateralen Halspartie bei Karotisdissektion, ipsilaterales HornerSyndrom zusätzlich zur N.-hypoglossus-Parese

- Vorerkrankungen: z. B. Erythema migrans als Hinweis auf eine Neuroborreliose

Diagnostischer „Workup“ Bei den 12 Hirnnerven stehen jeweils andere, zum Teil typische differenzialdiagnostische Überlegungen und Syndrome (z. B. A.-carotis-interna-Dissektion bei einseitiger Hypoglossusparese) im Vordergrund, weshalb die Läsionen der einzelnen Hirnnerven getrennt betrachtet werden.

Ursachen Wenn die „Ursache“ der Läsion nicht aufzuklären ist, wird sie oft als „idiopathisch“ bezeichnet. Bei der peripheren Fazialisparese hat das eine lange Tradition. Besser und eindeutiger ist: „Hirnnervenläsion $\mathrm{x} / \mathrm{y}$ rechts/links unklarer Ätiologie“. Verlaufskontrollen sollten dann geplant werden, da zu einem späteren Zeitpunkt eine Ursache erkennbar werden kann (z. B. Sarkoidose, kleinstes Neurinom, Meningeosis carcinomatosa etc.).

\section{Spezielle Fragestellungen}

In der folgenden Übersicht über alle Hirnnerven werden die häufigsten Ursachen einer Läsion aufgeführt [1]. Bei allen Hirnnerven gibt es „ungeklärte“ (,idiopathische“) Ursachen ( $\triangleright$ Tab. 1).

\section{N. olfactorius (I)}

Merke

Summe der Axone respiratorischer Rezeptorneurone, die in das Riechepithel eingebettet sind. Die Axone, von Schwann-Zellen umgeben, ziehen durch die Lamina cribrosa in die Bulbi olfactorii, die ein vorgeschobener Abschnitt des Frontallappens sind und zum ZNS gehören. 


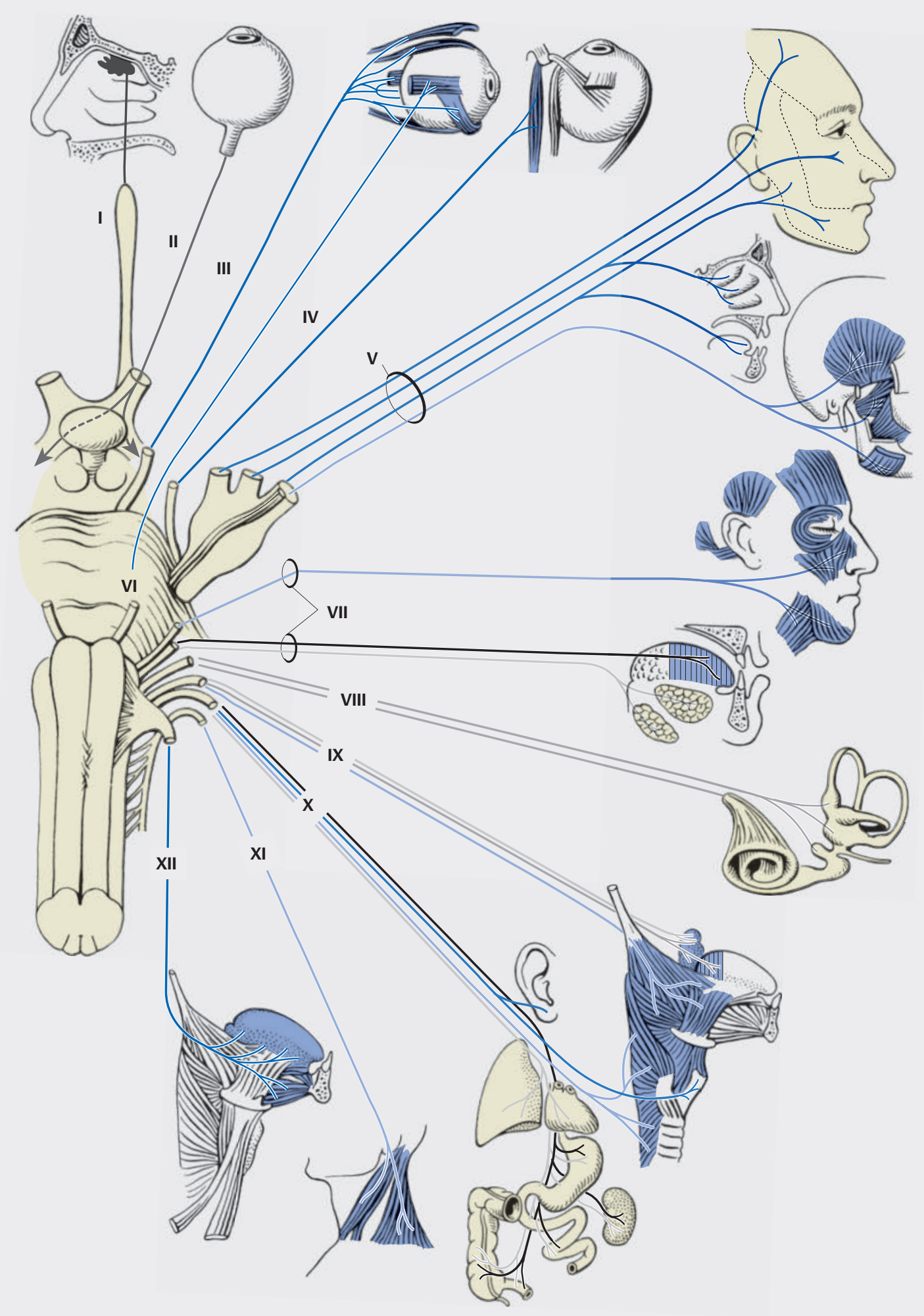

- Abb. 1 Hirnnerven: Austrittsstellen aus dem Hirnstamm, Bestandteile und Versorgungsgebiete. Quelle: Bähr M, Frotscher M. Neurologisch-topische Diagnostik. Stuttgart: Thieme; 2014 
Symptome Vermindertes oder ausgefallenes Geruchsempfinden (Hyposmie/Anosmie) für aromatische Geruchsstoffe.

\section{Diagnostik}

- Sniffin' Sticks Test - subjektive Untersuchungsmethode zur quantitativen psychophysischen Untersuchung von Riechstörungen

- Chemosensorisch evozierte Potenziale werden aufgrund des hohen apparativen Aufwandes nicht allgemein eingesetzt.

\section{Cave}

Diagnostische Fallgrube: Geruchsstörung bei neurodegenerativen Erkrankungen, Lewy-Körperchen-Erkrankung, Creutzfeldt-Jakob-Erkrankung, AlzheimerDemenz etc.

\section{Ursachen einer N.-olfactorius-Läsion}

- entzündlich

- Infektion: akute/chronische Infekte der oberen Atemwege (Rhinitis „postviral“, Sinusitis)

- systemische Erkrankung: z.B. endokrine Erkrankungen wie Diabetes mellitus, Nebenniereninsuffizienz (Morbus Addison), Hypothyreoidismus

- Creutzfeldt-Jakob-Erkrankung

- neurodegenerativ

- Morbus Parkinson, Demenz vom LewyKörperchen-Typ, Multisystematrophie

- Morbus Alzheimer

- Morbus Huntington

- Kompression/Infiltration

- Neoplasma (z. B. Meningeom)

- malnutritiv/toxisch

- Vitamin-A- und -B-Mangel

- Nieren- und Lebererkrankungen

- Toxine

- Medikamente: Antibiotika, Chemotherapeutika, Sympathomimetika, Antihypertensiva, Psychopharmaka, Antirheumatika

- Tab. 1 Sicherung der Läsion - diagnostisches Vorgehen.

\begin{tabular}{|c|c|c|c|c|c|}
\hline \multicolumn{3}{|c|}{ Hirnnerv } & Symptom & Test & Neurophysiologie \\
\hline I & \multicolumn{2}{|c|}{ N. olfactorius } & $\begin{array}{l}\text { Hypo-/Anosmie (aromatische } \\
\text { Stoffe) }\end{array}$ & $\begin{array}{l}\text { Sniff' Sticks } \\
\text { Test }\end{array}$ & $\begin{array}{l}\text { chemosensorisch evozierte } \\
\text { Potenziale }\end{array}$ \\
\hline ॥ & \multicolumn{2}{|c|}{ N. opticus } & Sehvermögen & $\begin{array}{l}\text { Sehtafeln, } \\
\text { Fundoskopie }\end{array}$ & VEP \\
\hline III & \multicolumn{2}{|c|}{ N. oculomotorius } & Ptose, Doppelbilder & \multirow[t]{3}{*}{ klinisch } & \multirow{3}{*}{$\begin{array}{l}\text { kein Instrument, } \\
\text { bei spezieller Kenntnis EMG } \\
\text { der Augenmuskeln }\end{array}$} \\
\hline IV & \multicolumn{2}{|c|}{ N. trochlearis } & Doppelbilder & & \\
\hline V & \multicolumn{2}{|c|}{ N. abducens } & Doppelbilder & & \\
\hline $\mathrm{VI}$ & \multicolumn{2}{|c|}{ N. trigeminus } & $\begin{array}{l}\text { Hypalgesie/Analgesie, } \\
\text { Parese Kaumuskeln }\end{array}$ & klinisch & $\begin{array}{l}\text { Blinkreflex, SEP, } \\
\text { Masseter-Silent-Period }\end{array}$ \\
\hline VII & \multicolumn{2}{|l|}{ N. facialis } & $\begin{array}{l}\text { Parese d. mimischen Muskeln, } \\
\text { ipsilateral Geschmacksausfall, } \\
\text { verminderte Tränensekretion }\end{array}$ & klinisch & $\begin{array}{l}\text { Blinkreflex, EMG, MEP, } \\
\text { Neurografie }\end{array}$ \\
\hline VIII & \multicolumn{2}{|c|}{ N. vestibulocholearis } & $\begin{array}{l}\text { Hypakusis/Anakusis, } \\
\text { Drehschwindel, } \\
\text { vegetative Symptome, } \\
\text { Nystagmus, } \\
\text { Fallneigung }\end{array}$ & $\begin{array}{l}\text { kalorische } \\
\text { Prüfung }\end{array}$ & AEP, Nystagmografie \\
\hline IX & \multicolumn{2}{|c|}{ N. glossopharyngeus } & $\begin{array}{l}\text { Berührungsempfinden } \\
\text { gestört im oberen Pharynx, } \\
\text { Würgereflex }\end{array}$ & $\begin{array}{l}\text { Video-Schluck- } \\
\text { Diagnostik }\end{array}$ & kein Instrument \\
\hline \multirow[t]{3}{*}{$x$} & \multirow[t]{3}{*}{ N. vagus } & Rr. pharyngei & Gaumensegel-Würgereflex & \multirow{2}{*}{$\begin{array}{l}\text { Video-Schluck- } \\
\text { Diagnostik }\end{array}$} & \multirow{2}{*}{$\begin{array}{l}\text { kein Instrument, } \\
\text { bei spezieller Kenntnis EMG }\end{array}$} \\
\hline & & Rr. laryngeus inferior (recurrens) & Stimmstörungen & & \\
\hline & & parasympathische Fasern & Herz-Kreislauf-Steuerung & $\begin{array}{l}\text { Herzfrequenz- } \\
\text { variabilität }\end{array}$ & $\begin{array}{l}\text { neurovegetative } \\
\text { Diagnostik }\end{array}$ \\
\hline $\mathrm{XI}$ & \multicolumn{2}{|c|}{ N. accessorius } & $\begin{array}{l}\text { Parese } \\
\text { M. sternocleidomastoideus }\end{array}$ & klinisch & Neurografie, EMG \\
\hline XII & \multicolumn{2}{|c|}{ N. hypoglossus } & $\begin{array}{l}\text { Abweichen der Zunge zur } \\
\text { paretischen Seite }\end{array}$ & klinisch & EMG \\
\hline
\end{tabular}


- hereditär

- kongenitale Anosmie: z.B. Kallmann-Syndrom (Anosmie und hypogonadotroper Hypogonadismus)

- traumatisch

- Schädelbasisfraktur, Contrecoup-Verletzungen mit okzipitalem Anprall

\section{N. opticus (II)}

Definition Bestandteil des ZNS. Die Axone der retinalen Ganglienzellen sind von Myelin aus Oligodendrozyten umgeben, weshalb der N. opticus bei einer Multiplen Sklerose betroffen sein kann. Der N. opticus ist von allen 3 Hirnhäuten und einem Liquorsaum umgeben. Hirndruckerhöhungen (chronische) können so unmittelbar druckschädigend auf den Sehnerv wirken.

\section{Symptome}

- vermindertes oder vollständig aufgehobenes Sehvermögen (Visus) eines Auges

\section{Diagnostik}

- Bestimmung des Nahvisus

- ophthalmoskopische Funduskopie (Stauungspapille, Papillitis, abgeblasste Papille bei Optikusatrophie)

- fingerperimetrische Gesichtsfelduntersuchung im Konfrontationstest

- Orbitasonografie mit Bestimmung der Papillenprominenz und des Durchmessers der Optikusscheide

- MRT - differenzierte Darstellung des N. opticus im gesamten Verlauf

- CT - knöcherne Verletzungen.

- visuell evozierte Potenziale (VEP)

Ziel der elektrophysiologischen Diagnostik

- VEP: Objektivierung und Differenzierung einer akuten vs. abgelaufenen Optikusneuritis

\section{Cave}

Diagnostische Fallgrube: Retinale Erkrankung (z. B. Vaskulitis der Retina beim Susac-Syndrom) als Differenzialdiagnose zur Optikusneuritis.

\section{Ursachen einer N.-opticus-Läsion}

- entzündlich

- immunologisch: Neuritis nervi optici bei Multipler Sklerose, Neuromyelitis optica

- Infektion: z. B. Borreliose, Toxoplasmose, Tbc, Lues, Denguefieber

- systemische Erkrankung: z. B. Sarkoidose, systemischer Lupus erythematodes, Morbus Wegener

- postvakzinal

- ischämisch

- arteriitische anteriore ischämische Optikusneuropathie: z. B. Riesenzellarteriitis

- nicht arteriitische anteriore ischämische Optikusneuropathie

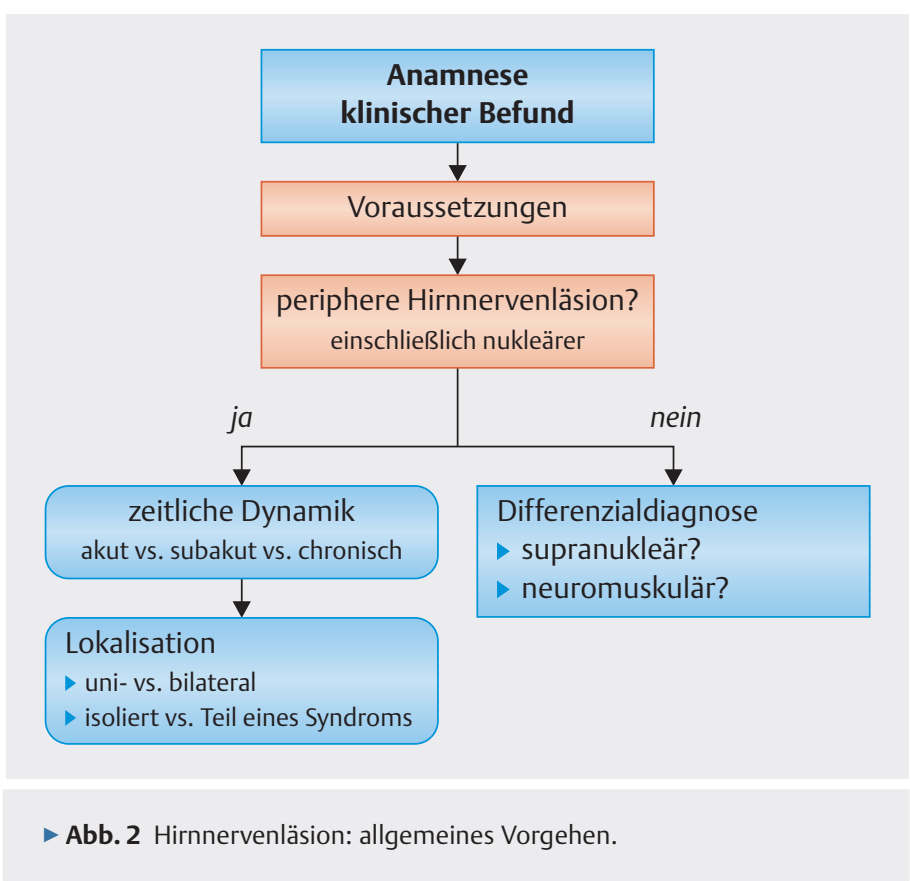

- Kompression/Infiltration

- Neoplasma: z. B. Optikusgliom, Optikusscheidenmeningeom

- Entzündung: z. B. endokrine Orbitopathie

- vaskulär: z.B. kavernöses Hämangiom

- Stauungspapille: erhöhter intrakranieller Druck

- Glaukom: erhöhter Augeninnendruck

- malnutritiv/toxisch/medikamentös

- z.B. Tabak-Alkohol-Amblyopie, Vitamin-B $12-M a n-$ gel

- z.B. nach Wespen-/Bienenstich, Methanol, Blei, Thallium, Arsen

- z.B. unter Adalimumab, Ethambutol, Isoniazid, Amiodaron, Tamoxifen, Disulfiram, Sildenafil

- hereditär

- z.B. hereditäre Leber-Optikusneuropathie, autosomale-dominante Optikusatrophie

- traumatisch

- Schädelbasisfraktur

\section{N. oculomotorius, N. trochlearis und N. abducens (N. III, IV, VI)}

Definition Die okulomotorischen Hirnnerven, N. oculomotorius (N. III), N. trochlearis (N. IV), N. abducens (N. VI), innervieren die 6 extraokulären Muskeln, die das Auge im dreidimensionalen Raum bewegen.

\section{Symptome}

- N. oculomotorius (N. III) - Ptosis und Augenfehlstellung mit Bulbusabweichung nach lateral mit übereinanderstehenden und schräg versetzten Doppelbildern. Bei Läsion der parasympathischen Anteile besteht eine zusätzliche Mydriasis. 
- N. trochlearis (N. IV) - vertikale Bulbusdivergenz, Angabe schräg versetzter Doppelbilder beim Blick nach unten

- N. abducens (N. VI) - Einwärtsschielen in Primärposition, Angabe horizontal stehender Doppelbilder, deren Abstand sich beim Blick in Richtung des paretischen Muskels vergrößert

\section{Diagnostik}

- Orbita-MRT - Darstellung der Muskeln - Dicke und Signalintensität vor/nach Kontrastmittel

- Elektromyografie einzelner Augenmuskeln ist technisch anspruchsvoll und potenziell komplikationsbehaftet.

Ziel der elektrophysiologischen Diagnostik Lokalisation der Läsion: Ein pathologischer Masseterreflex gibt Hinweis auf eine Hirnstammläsion (topologische Nähe des N. trigeminus zu den okulomotorischen Hirnnerven im Hirnstamm).

\section{Cave}

Diagnostische Fallgrube:

- neuromuskuläre Übertragungsstörungen bei okulärer Myasthenie/Lambert-Eaton-Syndrom/ Botulismus

- Myopathie der extraokulären Muskeln (z. B. okuläre Myositis, endokrine Orbitopathie)

- mesenzephaler Infarkt

\section{Ursachen einer Läsion der Hirnnerven III, IV und VI \\ - entzündlich}

- Infektion, z. B. basale Meningitis durch Tbc und Lues; Borreliose, HIV

- Varizella-Zoster-Virus-Reaktivierung (VZV-Reaktivierung) bei Zoster ophthalmicus

- systemisch: Sarkoidose, Kollagenosen (z.B. Sjögren-Syndrom); meist bilaterale Paresen beim Guillain-Barré-Syndrom und Miller-Fisher-Syndrom

- ischämisch

- diabetische Okulomotoriusparese (meist schmerzhaft unter Aussparung der Pupillomotorik)

- Riesenzellarteriitis

- Kompression/Infiltration

- Neoplasma: z. B. Neurinom, Hypophysenadenom, Kraniopharyngeom, paraselläres Meningeom, Nasopharyngealkarzinom, Epidermoid, Metastasen, multiples Myelom etc.

- Meningeosis carcinomatosa

- Entzündung: z. B. Sinusitis sphenoidalis

- vaskulär: N.-III-Läsion: z.B. Aneurysma der A. communicans posterior oder seltener der A. carotis interna (paralytisches Aneurysma), Karotisdissektion, Karotis-Sinus-cavernosus-Fistel, Sinus-cavernosus-Thrombose
- Druckerhöhung durch obere Herniation bei raumfordernden supratentoriellen Prozessen (v.a. N. III), Hirnvenenthrombose, idiopathische intrakranielle Hypertension

- Traktion

- Liquorunterdrucksyndrom (N. VI)

- traumatisch

- Orbitafraktur, Schädelbasisfraktur, Schädel-HirnTrauma (SHT)

- nukleär/faszikulär (Hirnstamm)

- Infarkt, Blutung, Multiple Sklerose, Tumor (mesenzephal: N. III, IV; pontin: N. VI)

- malnutritiv/toxisch

- Wernicke-Enzephalopathie bei Vitamin-B $B_{1}$-Mangel

- N.-VI-Parese nach Vincristin

- hereditär

- N. III: kongenitale Okulomotoriusparese

- N. IV: kongenitale Trochlearisparese

- N. VI: z. B. Duane-Syndrom

- Migräne

- ophthalmoplegische Migräne

- Tolosa-Hunt-Syndrom

- einseitiger orbitaler Dauerschmerz mit Ausfällen des N. III, IV, $\mathrm{V}_{1}$, VI

\section{N. trigeminus (V)}

\section{Definition}

- sensibel: Gesichtshaut, Scheitel-Ohr-Kinn-Linie

- motorisch: Kaumuskeln (Mm. masseter und temporalis) und Mundöffner (Mm. pterygoidei, M. geniohyoideus, vorderer Anteil des M. digastricus)

Symptome Hypästhesie/Hypalgesie im Versorgungsgebiet der Trigeminusäste und Parese der Kaumuskulatur. Nicht traumatische periphere Läsionen des N. trigeminus betreffen selten motorische Anteile mit Parese der Kaumuskulatur. Häufiger sind sensible Läsionen.

\section{Diagnostik}

- Blinkreflex - sensibler $\mathrm{V}_{1}$

- somatosensorisch evozierte Potenziale (SEP) - sensibel $\mathrm{V}_{2 / 3}$

- Masseter-Silent-Period - sensibel/motorisch $V_{3}$

- EMG - motorische Funktion von $\mathrm{V}_{3}-\mathrm{M}$. masseter; Nadelinsertion erfolgt im kaudalen Anteil über der Mandibula. Achtung: A. facialis (Palpation). M. temporalis; Nadelinsertion an der Schläfe. Achtung: A. temporalis superficialis (Palpation)

Ziel der elektrophysiologischen Diagnostik Objektivierung und Lokalisation der Läsion

Cave

Diagnostische Fallgrube: Sensibilitätsstörung im Trigeminusgebiet durch Läsion in der „Root Entry Zone“, z. B. pontiner Herd einer Multiplen Sklerose. 
- entzündlich

- Infektion, z.B. basale Meningitis durch Tbc, Lues; Borreliose, HIV

- VZV-Reaktivierung bei Zoster ophthalmicus

- systemisch: Sarkoidose, Morbus Wegener, SjögrenSyndrom, Guillain-Barré-Syndrom (GBS), Miller-Fisher-Syndrome (MFS)

- Kompression/Infiltration

- Neoplasma (z.B. Neurinom, Hypophysenadenom, Metastasen, Plasmozytom, Amyloidose etc.)

- Meningeosis carcinomatosa

- Histiozytose (Erdheim-Chester-Erkrankung)

- Reizsyndrom

- Trigeminusneuralgie: meist neurovaskuläres Kompressionssyndrom

- traumatisch

- Schädelbasis- und Mittelgesichtsfrakturen, iatrogen nach zahnärztlichen Eingriffen

- nukleär/faszikulär (Hirnstamm)

- pontomedulläre Läsion: Infarkt, Blutung, Multiple Sklerose, Gliom, Syringobulbie, Enzephalitis

- malnutritiv/toxisch

- z.B. Trichlorethylen

- hereditär

- kongenitale trigeminale Analgesie

- Tolosa-Hunt-Syndrom

- einseitiger orbitaler Dauerschmerz mit Ausfällen des N. III, IV, $\mathrm{V}_{1}$, VI

- FOSMN-Syndrom (Facial Onset sensory and motor Neuropathy)

- ätiologisch noch ungeklärt, evtl. Motoneuronvariante

\section{N. facialis (VII)}

Definition Motorische Fasern zur mimischen Muskulatur, beigemischt Chorda tympani (Geschmacksempfinden der vorderen $2 / 3$ der Zunge) und $N$. petrosus superficialis (Tränensekretion).

Symptome Parese der mimischen Muskulatur sowie gelegentlich begleitende ipsilaterale Geschmacksstörung und verminderte Tränensekretion.

\section{Diagnostik}

- transkranielle Magnetstimulation - proximaler N. facialis mit parietookzipitaler Spulenposition - kanalikulärer Läsionsort - zur Abgrenzung einer nukleären oder faszikulären Läsion im Hirnstamm. Hinweis: Eine kanalikuläre Untererregbarkeit auch der kontralateralen, klinisch nicht betroffenen Seite tritt zum Beispiel beim GBS oder einer Borreliose auf und trägt zur Abgrenzung von einer „Bell'schen Parese“ bei. Die kortikale Stimulation des Motorkortex kann eine supranukleäre Läsion belegen.
- Fazialiserregbarkeitstest - nach 2-3 Wochen Beurteilung der Waller'schen Degeneration zur Prognose (supramaximale elektrische Stimulation des N. facialis am Foramen stylomastoideum)

- Blinkreflexlokalisation (pathologische R2 bei medullären Prozessen) - Prognose (R1 erhalten: günstige Prognose)

- Nadelelektromyografie - hohe Entladungsrate der MUAP (Aktionspotenziale motorischer Einheiten motoric unit action potential) bei supranukleärer Parese, normal bei infranukleärer Parese

\section{Ziel der elektrophysiologischen Diagnostik}

- Differenzierung von pseudo-peripher-neurogenen Läsionen: z. B. Fazialis-MEP (motorisch evoziertes Potenzial) zur Differenzierung supranukleäre vs. infranukleäre/kanalikuläre Läsion

- Prognose - elektrische mastoidale Fazialisreizung und EMG. Bei supramaximaler mastoidaler Reizung spricht eine Minderung der Amplitude des Muskelaktionspotenzials (MAP) im Seitenvergleich nach 10-14 Tagen von weniger als $90 \%$ für eine günstige Prognose. Eine Amplitudendifferenz gegenüber der gesunden Seite von mehr als $90 \%$ hat bei $50 \%$ der Patienten eine ungünstige Prognose mit Restparesen und einer Defektheilung mit mehr oder weniger ausgeprägten Synkinesien. Verlaufsuntersuchung - Amplitudendifferenz bis $50 \%$ ist bei einmaliger Messung aufgrund der ausgeprägten inter- und intraindividuellen Variabilität nicht bewertbar.

- EMG-nachweisbare Reinnervationspotenziale sprechen für eine günstige Prognose, Persistenz pathologischer Spontanaktivität und fehlende Willküraktivität für eine ungünstige Prognose.

\section{Cave}

Diagnostische Fallgrube: zentrale Parese aufgrund eines kortikalen Infarkts mit Mundastschwäche als Differenzialdiagnose einer inkompletten peripheren Fazialisparese.

\section{Ursachen einer N.-facialis-Läsion}

- entzündlich

- Bell'sche Parese (idiopathische Parese) - wahrscheinlich v.a. durch eine Herpes-simplex-VirusReaktivierung

- Infektion z. B. Borreliose, HIV, Tbc, Lues, Otitis media

- VZV-Reaktivierung bei Zoster oticus (RamseyHunt-Syndrom)

- systemisch: Sarkoidose, Morbus Wegener, SjögrenSyndrom, GBS, MFS

- Melkersson-Rosenthal-Syndrom

- Kompression/Infiltration

- Neoplasma: z.B. Parotistumoren, Akustikusneurinom (=Vestibularisschwannom), Meningeom, Cholesteatom, Glomustumor 
- Meningeosis carcinomatosa

- Histiozytose (Erdheim-Chester-Erkrankung)

- geburtstraumatische Fazialisparese

- Reizsyndrom

- Spasmus hemifacialis: meist neurovaskuläres Kompressionssyndrom

- Fazialismyokymien: durch Hirnstammläsion oder peripher (z. B. Neuromyotonie)

- metabolisch

- Diabetes mellitus, Schwangerschaft

- traumatisch

- Felsenbeinfraktur

- nukleär/faszikulär (Hirnstamm)

- pontomedulläre Läsion: Infarkt, Blutung, Multiple Sklerose, Gliom, Syringobulbie, Enzephalitis

- hereditär

- z.B. Brown-Vialetto-Van-Laere-Syndrom, MöbiusSyndrom (= kongenitale doppelseitige Fazialis- und Abduzensparese)

- FOSMN-Syndrom

- ätiologisch noch ungeklärt, evtl. Motoneuronvariante

\section{N. vestibulocochlearis (VIII)}

Definition Afferenzen von den Bogengangsrezeptoren und Otolithen und Sinneszellen der Cochlea.

Symptome Läsion des cochleären Anteils führt zu Hypakusis bzw. Anakusis. Läsion des vestibulären Anteils (akut) bewirkt ein peripher vestibuläres Syndrom mit Drehschwindel, vegetativer Symptomatik, richtungsbestimmtem Spontannystagmus nach kontralateral sowie Fallneigung nach ipsilateral.

\section{Diagnostik}

- Audiometrie - Funktion der Pars cochlearis; Voraussetzung: intakte Schallleitung und Funktion der Hörknöchelchenkette

- akustisch evozierte Potenziale (AEP)

- Elektronystagmografie - Funktionsprüfung der Pars vestibularis mit kalorischer Untersuchung und Elektronystagmografie

Cave

Diagnostische Fallgrube: Pseudoneuritis vestibularis, z. B. durch einen Infarkt im Vestibulariskerngebiet als Differenzialdiagnose einer Neuritis vestibularis.

\section{Ursachen einer N.-vestibulocochlearis-Läsion \\ - entzündlich}

- Neuritis nervi vestibularis (akuter einseitiger partieller Vestibularisausfall): wahrscheinlich meistens durch eine Herpes-simplex-Virus-Reaktivierung

- Infektion z. B. Borreliose, HIV, Tbc, Lues, Otitis media
- VZV-Reaktivierung bei Zoster oticus (RamseyHunt-Syndrom)

- systemisch: Sarkoidose, Morbus Wegener

- Kompression/Infiltration

- Neoplasma: z. B. Akustikusneurinom (=Vestibularisschwannom)

- Meningeosis carcinomatosa

- Reizsyndrom

- Vestibularisparoxysmie und paroxysmaler Tinnitus: meist neurovaskuläres Kompressionssyndrom

- traumatisch

- Felsenbeinfraktur

- nukleär/faszikulär (Hirnstamm)

- pontomedulläre Läsion: Infarkt, Blutung, Multiple Sklerose, Gliom, Syringobulbie, Enzephalitis

- malnutritiv/toxisch:

- bilaterale Vestibulopathie, meistens durch ototoxische Substanzen (Aminoglykosidantibiotika, Chemotherapeutika etc.)

- Hämosiderosis cerebri

- hereditär

- z.B. Brown-Vialetto-Van-Laere-Syndrom

- Migräne

- vestibuläre Migräne

\section{N. glossopharyngeus (IX)}

Definition Motorische Fasern innervieren den M. stylopharyngeus und die oberen Anteile des M. cricopharyngeus. Sensible Fasern innervieren die Schleimhaut des hinteren Drittels der Zunge, der Tonsillen, des Nasopharynx, der Unterseite des weichen Gaumens einschließlich der Gaumenbögen, der eustachischen Tube und der Paukenhöhle. Sensorische Fasern versorgen die Geschmacksknospen des hinteren Drittels der Zunge.

Symptome Verminderte oder aufgehobene Berührungsempfindung im oberen Pharynxanteil, am Zungengrund und der Tonsillen. Würgereflex durch die Afferenzstörung bei streng einseitiger Auslösung abgeschwächt oder ausgefallen.

Diagnostik Der N. glossopharyngeus (IX) ist keiner apparativen Funktionsdiagnostik zugänglich.

\section{Ursachen einer N.-glossopharyngeus-Läsion}

- entzündlich

- Infektion, z. B. Borreliose, HIV, Tbc, Lues

- systemisch: Sarkoidose, Morbus Wegener, Amyloidose, GBS

- Kompression/Infiltration

- Neoplasma: Schädelbasistumor, Glomus-jugulareTumor, Epidermoid, Schwannome, Chordom (oft als Foramen-jugulare-Syndrom)

- Meningeosis carcinomatosa

- Aneurysma und Dissektion der A. carotis interna 
- Reizsyndrom

- Glossopharyngeusneuralgie: neurovaskuläres Kompressionssyndrom oder Eagle-Syndrom (zu langer Processus styloideus)

- traumatisch/iatrogen

- Schädelbasisfraktur, iatrogen bei Neck-Dissection und Tonsillektomien

- nukleär/faszikulär (Hirnstamm)

- pontomedulläre Läsion: Infarkt, Blutung, Multiple Sklerose, Gliom, Syringobulbie, Enzephalitis

\section{N. vagus $(X)$}

Definition Setzt sich aus motorischen, sensorischen, sensiblen und sekretorischen Anteilen zusammen.

Symptome Läsion der Rr. pharyngei - willkürliche und reflektorische Gaumensegelhebung. Bei einseitiger Läsion des N. vagus oder der Rr. pharyngei ipsilaterales Herabhängen des weichen Gaumens und eine ungenügende oder fehlende Hebung bei Phonation („a“) und beim Würgen. Beim Auslösen des Würgereflexes (efferenter Schenkel des Würgereflexes) oder beim Phonieren verzieht sich die hintere Pharynxwand zur gesunden Seite (Vorhang- oder Kulissenphänomen). Eine deutliche Parese führt beim Schlucken zu einer Regurgitation mit Flüssigkeitsaustritt aus der Nase.

\section{Diagnostik}

- Laryngoskopie - Läsion des N. laryngeus inferior (recurrens) Abweichen der Stimmritze von der Intermediärstellung

- EMG - Bestätigung einer neurogenen Läsion der Kehlkopfmuskulatur entweder lupenendoskopisch (M. cricoarytaenoideus) oder von außen (M. thyroarytaenoideus). Die Untersuchung des M. cricothyroideus ist technisch einfacher - Nadelinsertion knapp neben der Mittellinie oberhalb der tastbaren Rinne zwischen Ring- und Schildknorpel.

- Herzfrequenzvariabilität beim Valsalva-Manöver Funktionsstörung der parasympathischen Anteile des N. vagus

- Bulbusdruck-, Karotissinusdruck- und Gesichtseintauchversuch - autonome Funktionsstörungen des N. vagus

\section{Ursachen einer N.-vagus-Läsion}

- entzündlich

- Infektion z. B. Borreliose, HIV, Tbc, Lues,

- systemisch: Sarkoidose, Morbus Wegener, Amyloidose, GBS

- neurodegenerativ

- Morbus Parkinson, Demenz vom Lewy-Körperchen-Typ

- Kompression/Infiltration

- Neoplasma: Schädelbasistumor, Glomus-jugulareTumor, Epidermoid, Schwannome, Chordom (oft als Foramen-jugulare-Syndrom), weiter distal den
N. recurrens betreffend: Karzinome der Schilddrüse, Ösophagus, Mediastinum, Lunge

- Meningeosis carcinomatosa

- Aneurysma und Dissektion der A. carotis interna

- Reizsyndrom

- Gaumensegelmyoklonus (= Gaumensegeltremor) durch Läsion im Bereich des Guillain-Mollaret-Dreiecks im Hirnstamm

- Neuralgie des N. laryngeus superior durch eine neurovaskuläre Kompression

- traumatisch/iatrogen

- Schädelbasisfraktur, iatrogene Läsion des N. recurrens bei Schilddrüsenoperationen, Karotis-Thrombendarteriektomie (TEA)

- nukleär/faszikulär (Hirnstamm)

- pontomedulläre Läsion: Infarkt, Blutung, Gliom, Syringobulbie, Enzephalitis

\section{N. accessorius $(\mathrm{XI})$}

Definition Rein motorischer Nerv, der den M. trapezius und $\mathrm{M}$. sternocleidomastoideus versorgt.

Symptome Einseitige Accessoriusparese führt zur Lähmung des M. sternocleidomastoideus, die Drehung des Kopfes zur Gegenseite ist geschwächt und ggf. Atrophie des Muskels. Parese des M. trapezius - die betroffene Schulter steht etwas tiefer.

\section{Diagnostik}

- Neurografie - N. accessorius elektrische Stimulation dorsal des M. sternocleidomastoideus in Höhe des Larynx. Im Seitenvergleich werden die distal motorische Latenz und die Amplitude der M-Antwort des M. trapezius bewertet.

- EMG-Sicherung einer peripher neurogenen Läsion des $\mathrm{N}$. accessorius ist prognostisch (Reinnervationszeichen) hilfreich.

\section{Ursachen einer N.-accessorius-Läsion}

- entzündlich

- Infektion z. B. Borreliose, HIV, Tbc, Lues

- systemisch: Sarkoidose, Morbus Wegener, Amyloidose, GBS

- Kompression/Infiltration

- Neoplasma: Schädelbasistumor, Glomus-jugulareTumor, Epidermoid, Schwannome, Chordom (oft als Foramen-jugulare-Syndrom)

- Meningeosis carcinomatosa

- traumatisch/iatrogen

- Schädelbasisfraktur, iatrogene Läsion im Bereich des seitlichen Halsdreiecks bei diagnostischer Lymphknotenexstirpation, Neck-Dissection

- nukleär/faszikulär (Hirnstamm)

- pontomedulläre Läsion: Infarkt, Blutung, Gliom, Syringobulbie, Enzephalitis

- Motoneuronerkrankung: Amyotrophe Lateralsklerose, Morbus Kennedy 


\section{N. hypoglossus (XII)}

Definition Ausschließlich motorische Funktion, innerviert ipsilaterale Hälfte der Zungenmuskulatur.

Symptome Abweichen der Zunge nach Herausstrecken immer zur paretischen und atrophierten Seite hin, der intakte M. genioglossus schiebt die Zunge nach vorn. Seitwärtsbewegungen der Zunge geschehen nach der paretischen Seite nicht oder nur sehr unvollständig. Die Zunge ist auf der gelähmten Seite schmaler und stärker gefältelt.

Diagnostik EMG - Bestätigung einer peripheren Läsion. Möglichst entspannte Zunge. Nadelinsertion in den Zungenrand bei herausgestreckter und mit Gazetupfer festgehaltener Zunge. Alternativ: Nadelinsertion durch die Mundbodenmuskulatur von unten (manchmal bessere Entspannung).

\section{Cave}

Diagnostische Fallgrube: Zentrale Parese aufgrund eines kortikalen Infarkts mit Zungendeviation als Differenzialdiagnose einer N.-hypoglossus-Läsion vor Einsetzen der Waller'schen Degeneration mit konsekutiver Atrophie der Zunge.

\section{Ursachen einer N.-hypoglossus-Läsion}

- entzündlich

- Infektion, z. B. Borreliose, HIV, Tbc, Lues,

- systemisch: Sarkoidose, Morbus Wegener, Amyloidose, GBS, Chronisch inflammatorisch demyelinisierende Polyradikuloneuropathie, Hereditäre Neuropathie mit Neigung zu Drucklähmungen

- Kompression/Infiltration

- Neoplasma: Schädelbasistumor, Cholesteatom, Meningeom, Glomus-jugulare-Tumor

- Meningeosis carcinomatosa

- Aneurysma und Dissektion der A. carotis interna (mit oder ohne Horner-Syndrom)

- Reizsyndrom

- Spasmus hemilingualis durch neurovaskuläre Kompression

- traumatisch/iatrogen

- Schädelbasisfraktur, iatrogene Läsion bei KarotisTEA, Tonsillektomie, Intubation, Beatmung mit Larynxmaske

- hereditär

- z. B. Brown-Vialetto-Van-Laere-Syndrom

- nukleär/faszikulär (Hirnstamm)

- pontomedulläre Läsion: Infarkt, Blutung, Multiple Sklerose, Gliom, Syringobulbie, Enzephalitis, Arnold-Chiari-Malformation

- Motoneuronerkrankung: ALS, Morbus Kennedy
Interessenkonflikt

Der Autor gibt an, dass kein Interessenkonflikt vorliegt.

Autorinnen/Autoren

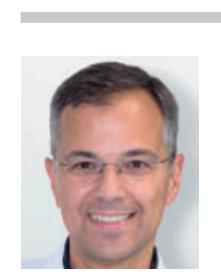

\section{Peter Paul Urban}

Prof. Dr. med., Facharztweiterbildung an der Neurologischen Klinik Universität Mainz; 1997-2006 dort Oberarzt. Seit 2006 Chefarzt der Neurologischen Abteilung Asklepios Klinik Barmbek, Hamburg. Akademisches Lehrkrankenhaus der Universität Hamburg und Medizinische Fakultät der Semmelweis-Universität Budapest (Campus Hamburg). 2007 Professur Universität Hamburg. Publikationen: 145 Originalarbeiten, 49 Buchbeiträge, 3 Bücher, 10 Wissenschaftspreise.

Korrespondenzadresse

Prof. Dr. med. Peter Paul Urban

Asklepios Klinik Barmbek

Rübenkamp 22

022291 Hamburg

p.urban@asklepios.com

\section{Erstveröffentlichung}

Dieser Beitrag wurde erstveröffentlicht in: Bischoff C, Buchner $\mathrm{H}, \mathrm{Hrsg}$. SOPs Klinische Neurophysiologie, 1. Aufl. Stuttgart: Thieme; 2018.

\section{Literatur}

[1] Hopf HC, Kömpf D. Erkrankungen der Hirnnerven. Stuttgart: Thieme; 2006

\section{Bibliografie}

DOI https://doi.org/10.1055/a-0655-8146

Neurologie up2date 2018; 1: 8-16

(c) Georg Thieme Verlag KG Stuttgart · New York ISSN 2511-3453 